\title{
Postmortem computed tomography of gas gangrene with aortic gas in a dialysis patient
}

\author{
Rin Asao $^{1} \cdot$ Kazumasa Nishida ${ }^{1} \cdot$ Hiromichi Goto ${ }^{1} \cdot$ Yoshikazu Goto $^{2} \cdot$ Noriatsu Ichiba $^{3} \cdot$ Isao Ohsawa $^{1}$
}

Received: 3 August 2019 / Accepted: 11 February 2020 / Published online: 22 April 2020

(c) The Author(s) 2020

\begin{abstract}
Recently, postmortem imaging is sometimes used as an alternative to conventional autopsy. However, there are few case reports of postmortem imaging of dialysis patients. Here, we report a fatal case of gas gangrene involving a 76-year-old man who underwent dialysis. He died suddenly before a diagnosis could be established. Immediately after his death, postmortem computed tomography (PMCT) revealed gas accumulation in his right upper extremity and ascending aorta. Gas gangrene progresses rapidly and may sometimes result in sudden death before it is diagnosed. In this case, PMCT findings were useful to diagnose gas gangrene. Intravascular gas is a common finding on PMCT and is generally caused by cardiopulmonary resuscitation and decomposition. However, the detection of gas in the ascending aorta by PMCT was not described previously. Moreover, Gram stain and culture of the exudate showed anaerobic Gram-positive bacilli which suggested that the gas generation in the blood was caused by Clostridia species. To the best our knowledge, this is the first report of a dialysis patient whose cause of death was determined as gas gangrene using PMCT.
\end{abstract}

Keywords Gas gangrene $\cdot$ Postmortem imaging $\cdot$ Dialysis $\cdot$ Intravascular gas $\cdot$ Clostridia species

\section{Introduction}

Gas gangrene is a necrotic infection of the soft tissue and muscles that progresses rapidly and it has high mortality rates. In approximately $80 \%$ of the cases, gas gangrene is caused by Clostridium perfringens which is a Gram-positive spore-forming bacillus commonly found in the soil and intestines of humans and animals [1,2]. Deep penetrating injuries compromise the blood supply and create an anaerobic environment that is ideal for spore germination and bacterial proliferation [3]. Other predisposing conditions are bowel and biliary tract surgery, intramuscular injection, gynecologic procedure, and intrauterine fetal death [1]. Although the clinical course of Clostridia gas gangrene is fulminant, patients do not usually exhibit specific or severe symptoms

Isao Ohsawa

i.osawa@saiyukai.com

1 Department of Nephrology, Saiyu Soka Hospital, 1-7-22 Matsubara, Soka, Saitama, Japan

2 Department of Neurosurgery, Saiyu Soka Hospital, Soka, Japan

3 Department of Radiology, Otsu City Hospital, Otsu, Japan at onset. In addition, early diagnosis of necrotizing infections may be confounded by other factors [1]. Crescendo pain is the most important clinical manifestation of such infections. However, because pain can be absent in patients receiving analgesic agents and those having diabetes-related neuropathy or altered mental status, patients sometimes die shortly before a definitive diagnosis is established [1].

Currently, postmortem imaging is sometimes used as an alternative to conventional autopsy. In Japan, approximately 40,000 conventional autopsies were performed every year in the 1980s. However, the reported number of conventional autopsies is gradually decreasing. In the 2010s, the number of conventional autopsies was slightly higher than 11,000 per year [4]. Postmortem imaging has a shorter examination time and is less invasive than conventional autopsy. Wagensveld et al. evaluated the frequency of total-body computed tomography (CT) and magnetic resonance imaging features of postmortem changes in in-hospital deaths and reported a wide variety of features of postmortem changes [5]. To investigate the accuracy of postmortem imaging, a few groups compared postmortem imaging with conventional autopsy and showed the accuracy of postmortem computed tomography (PMCT) [6, 7]. PMCT is useful in detecting gas accumulation inside the body which is an advantage over 
the conventional autopsy. Therefore, PMCT is expected for a diagnosis of gas gangrene. Recently, few reports described PMCT with gas gangrene. Yamaguchi et al. reported the first autopsy case of fatal septicemia caused by gas-forming C. perfringens using PMCT findings. They combined conventional autopsy and PMCT to investigate a cadaver which was presented to the hospital in a cardiopulmonary arrest state and died [8]. Kitano et al. reported a case of in-hospital death in which PMCT revealed sepsis to be the cause of death [9].

To the best of our knowledge, there are few reports which describe PMCT in dialysis patients. Here, we report a dialysis patient who died suddenly owing to gas gangrene. In this case, we determined the diagnosis by PMCT. Additionally, we detected an intriguing finding, aortic gas.

\section{Case report}

A 76-year-old man, who was diagnosed with type 2 diabetes mellitus (DM) 26 years ago, underwent dialysis for 9 years owing to diabetic nephropathy. He walked into a hospital at 22:00 p.m. with complaints of fever and abdominal discomfort. The medical history of the patient included cerebral infarction but not infection. He was awake and had a blood pressure of 189/94 $\mathrm{mmHg}$, pulse of 102 beats per minute, body temperature of $38.8^{\circ} \mathrm{C}$, and saturation of percutaneous oxygen of $98 \%$ at room air. Physical examination showed that he had upper abdominal tenderness with normal bowel sounds. He had left incomplete paralysis owing to prior cerebral infarction. He did not complain of pain in his extremities and no apparent injury was found, except for a vascular puncture site for a shunt in his left forearm. Laboratory test results revealed a mildly elevated leukocyte count $(11,600 / \mu \mathrm{L})$, and his DM was poorly controlled (Table 1$)$. The systemic inflammatory response syndrome score met two criteria (body temperature $>38^{\circ} \mathrm{C}$ and heart rate $>90 /$ min). X-ray, electrocardiogram, and chest and abdominal CT showed no remarkable alterations. Based on the physical examination and laboratory tests, enterogastritis was initially

Table 1 Laboratory findings on admission

\begin{tabular}{lllcll}
\hline WBC & $11,600 / \mu \mathrm{L}$ & AST & $13 \mathrm{U} / \mathrm{L}$ & $\mathrm{Na}$ & $139 \mathrm{mmol} / \mathrm{L}$ \\
$\mathrm{RBC}$ & $390 \times 10^{4} / \mu \mathrm{L}$ & ALT & $9 \mathrm{U} / \mathrm{L}$ & $\mathrm{K}$ & $4.2 \mathrm{mmol} / \mathrm{L}$ \\
$\mathrm{Hb}$ & $13.2 \mathrm{~g} / \mathrm{dL}$ & $\gamma \mathrm{GTP}$ & $17 \mathrm{U} / \mathrm{L}$ & $\mathrm{Cl}$ & $103 \mathrm{mmol} / \mathrm{L}$ \\
$\mathrm{Ht}$ & $39.40 \%$ & ALP & $192 \mathrm{U} / \mathrm{L}$ & $\mathrm{Ca}$ & $9.3 \mathrm{Mg} / \mathrm{dL}$ \\
$\mathrm{Plt}$ & $11.8 \times 10^{4} / \mu \mathrm{L}$ & $\mathrm{LDH}$ & $162 \mathrm{U} / \mathrm{L}$ & $\mathrm{HbA} 1 \mathrm{c}$ & $7.8 \%$ \\
$\mathrm{PT}$ & $11.7 \%$ & $\mathrm{CK}$ & $99 \mathrm{U} / \mathrm{L}$ & $\mathrm{CRP}$ & $0.17 \mathrm{mg} / \mathrm{dL}$ \\
APTT & $26.8 \mathrm{~s}$ & $\mathrm{~T}-\mathrm{Bil}$ & $0.7 \mathrm{mg} / \mathrm{dL}$ & \\
& & $\mathrm{BUN}$ & $46.1 \mathrm{mg} / \mathrm{dL}$ & \\
& & $\mathrm{Cr}$ & $8.22 \mathrm{mg} / \mathrm{dL}$ & \\
\hline
\end{tabular}

suspected. However, he complained that his pain worsened and experienced sudden swelling in his right upper extremity at 7:00 a.m. Despite cefazolin administration, he rapidly developed a state of shock and had a cardiopulmonary arrest at 7:50 a.m. Cardiac compression and artificial respiration with bag valve mask and respirator were performed for 30 min. Central venous catheterization was not performed. Despite attempts of resuscitation, his death was confirmed.

The cadaver had cutaneous emphysema and subcutaneous hemorrhage on the torso and right upper extremities (Fig. 1a-c). Moreover, the deceased patient developed skin blisters with a foul smell (Fig. 1d). Because his family refused a conventional autopsy, whole-body PMCT was performed within $70 \mathrm{~min}$ of his death. A 64-multidetectorrow CT (SOMATOM Perspective, Siemens Healthineers, Germany) scan was performed. The patient was scanned from the head to the pelvis in the supine position.

Although chest CT on admission showed no gas densities in the right upper extremity (Fig. 2a, b), PMCT showed remarkable gas densities in the subcutaneous and muscular layer of the right chest wall and upper extremity (Fig. 2c). Moreover, gas was observed even in the ascending aorta (Fig. 2d). A blood culture could not be performed owing to technical problems. A culture test result of the exudate from the skin bullae revealed a proliferation of Gram-positive bacilli. No aerobic bacteria were detected. We presumed that the cause of death was gas gangrene caused by Clostridia species.

\section{Discussion}

Gas gangrene consists of two major presentations: traumatic and spontaneous. Traumatic gas gangrene occurs after crushtype injury, penetrating injury, gynecologic surgery such as abortion, and bowel/biliary tract surgery where bowel contents leak into the soft tissues. Spontaneous gas gangrene generally occurs via hematogenous seeding of bacteria from the gastrointestinal tract. However, in approximately 20-30\% of the cases, the primary source of the infection is unknown $[10,11]$. In this case, because the patient had no apparent traumatic injury, except for a vascular access puncture site for a shunt in the left forearm, and initially complained of abdominal discomfort, the focus and route of this infection are unknown. A very small injury may have existed in his upper right extremity. Clostridia species, E. coli, Klebsiella pneumoniae, Aeromonas hydrophilia, etc. are known as pathogens that cause gas gangrene [1, 9, 11-13]. In this case, Gram staining of the exudate from the skin bullae showed Gram-positive bacilli. Thus, it was conceivable that the gas gangrene was caused by Clostridia species.

When interpreting PMCT images, normal postmortem changes should be recognized and distinguished from fatal 
Fig. 1 External appearance: a skin purpura and swelling on the torso and right upper extremity. b, c Cutaneous emphysema and subcutaneous hemorrhage on the right upper extremity. d Bubbles in skin blisters
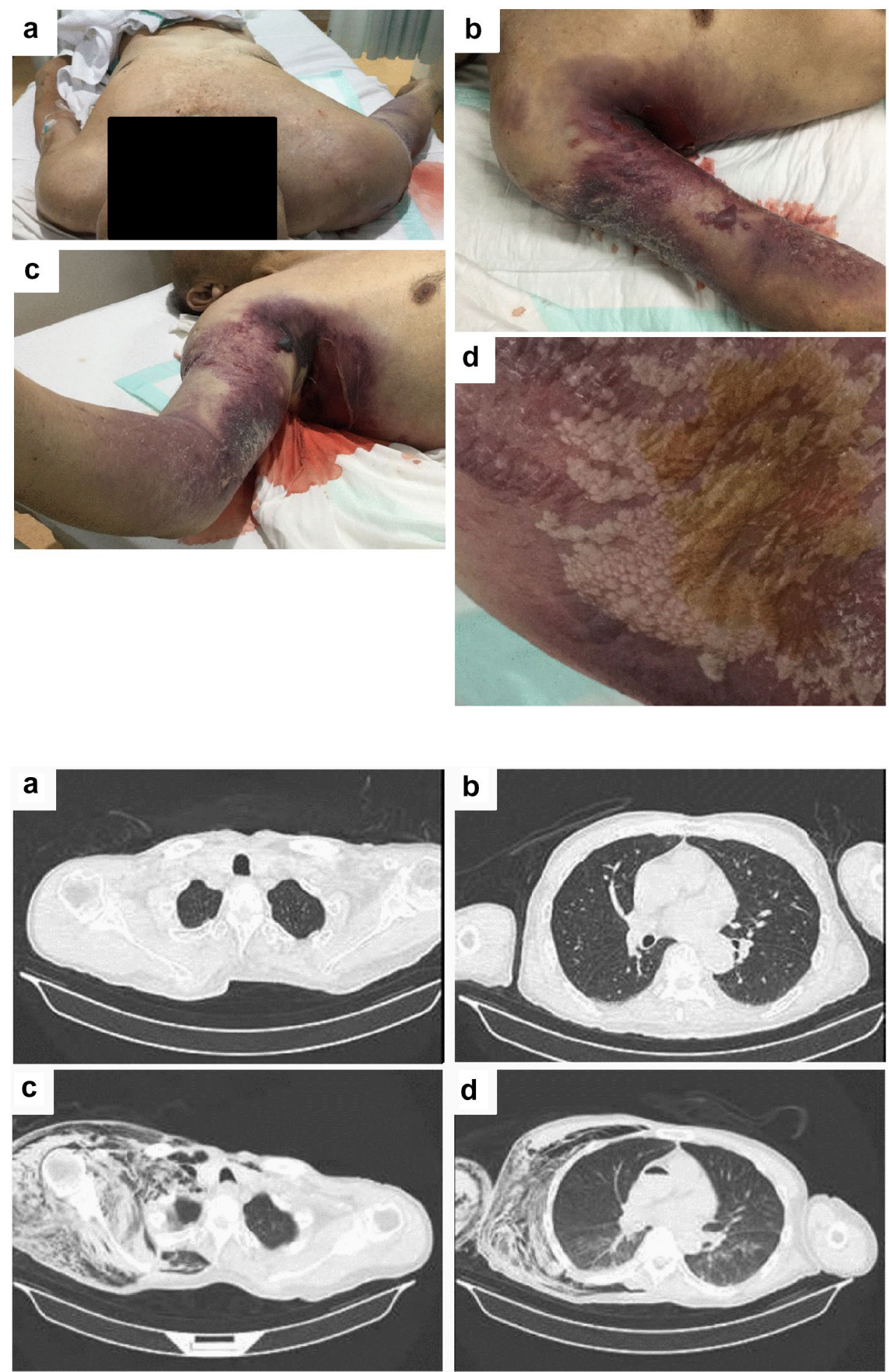

Fig. 2 Chest computed tomography images on admission (upper panels) and postmortem computed tomography images (lower panels): a no remarkable changes were observed in subcutaneous regions and muscles. b No remarkable changes were observed in lung and cardiovascular system. c A massive amount of gas was observed in the right upper extremity. d Accumulation of gas was observed both within the torso and in the aorta 
reach systemic circulation [17-19]. Cardiovascular gas was observed in $71 \%$ of the patients who underwent CPR and gas accumulation was observed in the right heart in most cases. However, there is no reported case of aortic gas development [17]. In this case, intravascular gas was observed not in the right heart but in the aorta. Okuda et al. reported the case of myocardial intravascular gas in the left ventricle on PMCT performed immediately after death certification [22]. As $\mathrm{CO}_{2}$ concentration in the right heart and myocardial vein increased because of low cardiac output, anaerobic metabolism progression, and decreased $\mathrm{CO}_{2}$ transport, the blood became supersaturated and released excess solute $\mathrm{CO}_{2}$ as gas bubbles after death [22]. Kitano et al. reported a case of death owing to sepsis without CPR. PMCT performed $2 \mathrm{~h}$ after death revealed intravascular gas [9]. They described that the cause of intravascular gas on PMCT was not only CPR and decomposition but also gas-producing bacterial sepsis [9]. In this case, PMCT was performed immediately after death certification, and intravascular gas was detected in the ascending aorta. Therefore, decomposition and CPR were less likely to be the cause of aortic gas than gas-forming bacteria. As Clostridia species produce large quantities of gas rapidly in an anaerobic condition, it was conceivable that aortic gas was produced by Clostridia species in this case.

This case report has some limitations. First, there was a lack of clinical data before the patient's condition suddenly worsened. Because this patient had poorly controlled diabetes and underwent dialysis, it is likely that he was immunocompromised. Thus, there was a high prevalence of infection in this case. Second, pictures of Gram staining of the exudate were not taken and anaerobic culture of exudate and blood culture was not performed, although these tests were necessary to identify the pathogen causing gas gangrene. However, Gram staining and culture of the exudate showed anaerobic Gram-positive bacilli which suggested that the responsible bacteria might be Clostridia species.

In conclusion, we report a gas gangrene case that was diagnosed based on clinical course and PMCT findings. To the best of our knowledge, this is the first report of a dialysis patient whose cause of death was determined as gas gangrene using PMCT. Gas gangrene progresses rapidly and may sometimes result in sudden death before a diagnosis is made. Moreover, aortic gas on the PMCT performed immediately after the death of a patient with septicemia is a key finding of this report.

Acknowledgements This manuscript was subjected to English proofreading by Editage.

Author contributions Clinical examination and acquisition of data: RA and KN. Analysis and interpretation of data: RA, NI and IO. Drafting of the manuscript: RA. Critical revision of the article for important intellectual content: NI and IO. Supervision: HG, KG, and IO.

\section{Compliance with ethical standards}

Conflict of interest The authors have declared that no conflict of interest exists.

Human and animal rights statement This article does not contain any studies with human participants or animals performed by any of the authors.

Informed consent Informed consent was obtained from the surviving family of the patient in this case report.

Open Access This article is licensed under a Creative Commons Attribution 4.0 International License, which permits use, sharing, adaptation, distribution and reproduction in any medium or format, as long as you give appropriate credit to the original author(s) and the source, provide a link to the Creative Commons licence, and indicate if changes were made. The images or other third party material in this article are included in the article's Creative Commons licence, unless indicated otherwise in a credit line to the material. If material is not included in the article's Creative Commons licence and your intended use is not permitted by statutory regulation or exceeds the permitted use, you will need to obtain permission directly from the copyright holder. To view a copy of this licence, visit http://creativecommons.org/licenses/by/4.0/.

\section{References}

1. Stevens DL, Bryant AE. Necrotizing soft-tissue infections. N Engl J Med. 2017;377(10):2253-65.

2. Stevens DL, Bryant AE. The role of Clostridial toxins in the pathogenesis of gas gangrene. Clin Infect Dis. 2002;35(Suppl 1):S93-100.

3. Bryant AE, Stevens DL. Clostridial myonecrosis: new insight in pathogenesis and management. Curr Infect Dis Rep. 2010;12:383-91.

4. Annual of pathological autopsy cases in Japan. Japanese Pathological Society. http://pathology.or.jp/kankoubutu/all_hyou.html. Accessed 6 Aug 2019

5. Wagensveld IM, Blokker BM, Wielopolski PA, et al. Total-body $\mathrm{CT}$ and MR features of postmortem change in in-hospital deaths. PLoS One. 2017;12:e0185115.

6. Roberts ISD, Benamore RE, Benbow EW, Lee SH, Harris JN, Jackson A, et al. Post-mortem imaging as an alternative to autopsy in the diagnosis of adult deaths: a validation study. Lancet. 2012;379(9811):136-42.

7. Grabherr S, Heinemann A, Vogel H, Rutty G, Morgan B, Wozniak $\mathrm{K}$, et al. Postmortem CT angiography compared with autopsy: a forensic multicenter study. Radiology. 2018;288(1):270-6.

8. Yamaguchi R, Makino Y, Chiba F, Motomura A, Inokuchi G, Yajima D, et al. Fatal Clostridium perfringens septicemia suggested by postmortem computed tomography: a medico-legal autopsy case report. Forensic Sci Int. 2015;253:e4-9.

9. Kitano F, Kinoshita K, Noriki S, Inai K. Postmortem intravascular gas caused by antemortem bacterial sepsis. Intern Med. 2019;58(3):457-8.

10. Simon TG, Bradley J, Jones A, Carino G. Massive intravascular hemolysis from Clostridium perfringens septicemia: a review. $\mathrm{J}$ Intensive Care Med. 2014;29(6):327-33.

11. van Bunderen CC, Bomers MK, Wesdorp E, Peerbooms P, Veenstra J. Clostridium perfringens septicaemia with massive 
intravascular haemolysis: a case report and review of the literature. Neth J Med. 2010;68(9):343-6.

12. Kershaw CJ, Bulstrode C. Gas gangrene in a diabetic after intramuscular injection. Postgrad Med J. 1988;64(756):812-3.

13. Furusu A, Yoshizuka N, Abe K, Sasaki O, Miyazaki K, Miyazaki M, Hirakata Y, Ozono Y, Harada T, Kohno S. Aeromonas hydrophila necrotizing fasciitis and gas gangrene in a diabetic patient on haemodialysis. Nephrol Dial Transplant. 1997;12(8):1730-4.

14. Takahashi N, Higuchi T, Shiotani M, Maeda H, Hirose Y. Intrahepatic gas at postmortem multislice computed tomography in cases of nontraumatic death. Jpn J Radiol. 2009;27(7):264-8.

15. Jackowski C, Sonnenschein M, Thali MJ, Aghayev E, Yen K, Dirnhofer R, Vock P. Intrahepatic gas at postmortem computed tomography: forensic experience as a potential guide for in vivo trauma imaging. J Trauma. 2007;62(4):979-88.

16. Asamura H, Ito M, Takayanagi K, Kobayashi K, Ota M, Fukushima H. Hepatic portal venous gas on postmortem CT scan. Leg Med (Tokyo). 2005;7(5):326-30.

17. Shiotani S, Kohno M, Ohashi N, Atake S, Yamazaki K, Nakayama H. Cardiovascular gas on non-traumatic postmortem computed tomography (PMCT): the influence of cardiopulmonary resuscitation. Radiat Med. 2005;23(4):225-9.
18. Yamaki T, Ando S, Ohta K, Kubota T, Kawasaki K, Hirama M. CT demonstration of massive cerebral air embolism from pulmonary barotrauma due to cardiopulmonary resuscitation. J Comput Assist Tomogr. 1989;13(2):313-5.

19. Hwang SL, Lieu AS, Lin CL, Liu GC, Howng SL, Kuo TH. Massive cerebral air embolism after cardiopulmonary resuscitation. $\mathrm{J}$ Clin Neurosci. 2005;12(4):468-74.

20. Sakata M, Miki A, Kazama H, Morita M, Yasoshima S. Studies on the composition of gases in the post-mortem body: animal experiments and two autopsy cases. Forensic Sci Int. 1980;15(1):19-29.

21. Ikels KG. Production of gas bubbles in fluids by tribonucleation. J Appl Physiol. 1970;28:524-7.

22. Okuda T, Shiotani S, Kobayashi T, Kohno M, Hayakawa H, Kikuchi K, Suwa K. Immediate non-traumatic postmortem computed tomographic demonstration of myocardial intravascular gas of the left ventricle: effects from cardiopulmonary resuscitation. SpringerPlus. 2013;2(1):86.

Publisher's Note Springer Nature remains neutral with regard to jurisdictional claims in published maps and institutional affiliations. 